\title{
«VIRTUOSI SENZA CUJONI!» \\ TRA ITALIANO, LATINO E TEDESCO NELLE LETTERE DI LUDWIG VAN BEETHOVEN
}

\author{
Debora de Fario ${ }^{1}$
}

\section{PREMESSA}

Se è ampiamente dimostrato quanto l'italiano fosse conosciuto ed usato come lingua della musica in numerosi paesi europei ${ }^{2}$, tra cui la Germania $^{3}$, forse non è altrettanto noto che il grande musicista Ludwig van Beethoven usasse espressioni della nostra lingua anche al di fuori dell'àmbito strettamente musicale 4 .

Tra la metà del Settecento e l'inizio dell'Ottocento, quando si colloca la sua vicenda umana, la nostra è ancora «una delle lingue internazionali di cultura» (Motolese, 2001: 440); non solo: «sotto gli Asburgo l'italiano è - accanto al latino e al tedesco - la terza lingua ufficiale» nella quale era possibile, tra gli altri usi, anche stendere documenti ed atti burocratici ${ }^{5}$.

${ }^{1}$ Università degli Studi della Basilicata.

Questo lavoro deve molto ai consigli e ai suggerimenti di Ilaria Bonomi, Maria Antonietta Epifani e Giorgio Marrapodi. La ricerca è nata nel curare con Maria Antonietta Epifani uno speciale per la sezione Lingua italiana di Treccani in occasione del $250^{\circ}$ dalla nascita (consultabile al sito

https://www.treccani.it/magazine/lingua_italiana/speciali/Beethoven/03_Epifani_de_Fazio.html).

${ }^{2}$ Cfr. Bonomi (2010: 185): «I termini musicali, è noto, sono nelle lingue europee, e non solo in queste, in gran parte di origine italiana, e rappresentano una percentuale molto consistente degli italianismi di queste lingue (900 su circa 4300)». Il dato numerico è desunto dalla studiosa dal lemmario del DIFIT (oggi disponibile anche online - difit.italianismi.org - all'interno del progetto Osservatorio degli italianismi nel mondo (OIM) che fa capo all'Accademia della Crusca. Lo stato degli studi sull'argomento (a partire dalla sollecitazione di Folena 1983) è tratteggiato abilmente da Bonomi che presenta un quadro in cui è prevalsa da parte degli studiosi soprattutto in passato più l'attenzione per l'aspetto "storico" (ossia la supremazia dell'italiano come lingua della poesia per musica; cfr. Bonomi (1998 e 2015) e Stammerjohann (1990); di qui anche il taglio dei principali strumenti lessicografici inerenti il lessico musicale: cfr. almeno il DEUMM e il LESMU) che per quello terminologico, il quale solo in seguito è stato oggetto di attenzione (cfr. Bonomi, 2010: 186 e segg.; Stammerjohann, 2013 e la bibliografia indicata).

3 Per la diffusione dell'italiano della musica in Germania, cfr. Bonomi (2010: 215-216); importante la presenza in tedesco di un ricco filone di dizionari di forestierismi, molto utile come fonte di voci musicali: cfr. Bonomi (2010: 216-218).

${ }_{4}^{4}$ Tra i quarantaquattro volumi che costituivano nel 1825 la sua biblioteca non di argomento musicale figura anche un vocabolario italiano-tedesco (Carli Ballola, 1977: 98). Inoltre egli frequentò occasionalmente (dal 1793 al 1802) Antonio Salieri «per averne consigli sul modo di trattare in mus[ica] la lingua italiana». (DEUMM 376; cfr. anche Pestelli, 1979: 229-30). Nel decennio indicato, Beethoven compose circa venticinque duetti, terzetti e quartetti tutti su testi di Metastasio e i brani «presentano frequenti correzioni di Salieri, soprattutto per ciò che riguarda la declamazione mus[icale] della lingua it[aliana] e il corretto impiego dell'elisione» (DEUMM 376).

${ }^{5}$ Per la peculiare percezione della nostra lingua nei paesi germanici, cfr. Stammerjohann (1997: 40) «l'italiano che imparavano i tedeschi era diverso da quello dei francesi e degli inglesi, era un italiano meno letterario e più pratico». Cfr. anche Motolese (2001: 440) che nota inoltre come i manuali di conversazione ad uso dei tedeschi abbiano «un carattere colloquiale, mercantile, economico e politico, fondato sull'uso, ben differente da quello dei manuali inglesi o francesi, che descrivono una lingua "salottiera" ». Su questo aspetto, cfr. anche Folena (1983: 436-37). 
L'argomento si colloca al crocevia di alcuni importanti settori di studi che riguardano l'italiano usato con intenti letterari e non letterari da scriventi non italofoni. Questo filone è stato iniziato dai lavori, a partire dagli anni Sessanta, di Gianfranco Folena sullitaliano di Voltaire e di Mozart (poi confluiti in Folena, 1983), che riguardavano proprio l'analisi degli epistolari dei due autori, a cui sono seguiti quelli di Furio Brugnolo sul plurilinguismo di carattere letterario ${ }^{6}$. Proprio il genere epistolare ha rappresentato tra Settecento e Ottocento $^{7}$ un importante banco di prova per l'italiano usato da scriventi non italiani. Oltre ai già citati Voltaire e Mozart, abbiamo le lettere di Byron (Cartago, 2002), Stendhal (Vitale, 1982; Meacci, 2001), Joyce (Melchiori, 1994). In questi epistolari, scrittori e artisti stranieri «piegano la nostra lingua alla loro personalissima scrittura, insofferente spesso dei nessi grammaticali» (Brugnolo, 2011: 1292) ${ }^{8}$ ed essa è caratterizzata ovviamente da vistosi fenomeni di contaminazione con la lingua d'origine e «tende a diventare quasi una lingua speciale da plasmare e manipolare» (Brugnolo, 2011: 1292) .

Abbiamo pertanto spogliato per intero l'ultima edizione delle lettere beethoveniane, curata dallo studioso tedesco Sieghard Brandenburg ${ }^{10}$.

Diciamo subito che nei Briefe beethoveniani l'italiano occupa quantitativamente un posto piuttosto marginale. Si tratta perlopiù infatti di piccole inserzioni - spesso collocate in punti strategicamente importanti nel tessuto della lettera - singole parole, ma rilevanti nella complessiva architettura delle stesse. Mentre numerose missive risultano scritte interamente in un fluente francese ${ }^{11}$, così come nella lingua d'oltralpe sono spesso non brevi inserti narrativi in quelle in tedesco ${ }^{12}$, nell'Epistolario risultano essere soltanto tre le

${ }^{6}$ Brugnolo (1983, 2009, 2010), Brugnolo-Orioles (2002). Brugnolo (2009: 11) ricorda il meritorio volume della Storia della letteratura italiana diretto da Enrico Malato dedicato proprio alla "Letteratura italiana fuori d'Italia" che si occupa in realtà solo marginalmente degli scrittori stranieri che hanno prodotto opere letterarie in italiano: «e gli unici autori di questo tipo su cui si sofferma a fondo sono Louise Labé, John Milton, James Joyce ed Ezra Pound» (su Joyce, cfr. anche Zanotti, 1999). In realtà la lista si allarga a dismisura (con produzioni eterogenee per genere, stile, tempo e provenienza geografica degli esecutori): il trovatore Raimbaut de Vaqueiras, gli spagnoli Carvajal, Aldana e Quevedo, i francesi Bricard, Montaigne e Régnier Desmarais, gli inglesi Byron (per cui cfr. Bruni, 1999), Shelley, Mathias e Rossetti, i greci Kalvos, Salomós e Sarandaris, e tanti altri oggetto del citato volume dello studioso.

${ }^{7}$ I soli precedenti seicenteschi sono rappresentati dai carteggi dei pittori fiamminghi Pietro Paolo Rubens (per cui cfr. Meacci, 2001: 445-446) e Jan Brueghel il vecchio (per cui. cfr. Argenziano, 2019).

8 Stile e lingua variano moltissimo da autore ad autore, anche in relazione col destinatario. In Voltaire (Folena, 1983) si spazia dalle missive alla nipote-amante Marie-Louise Denis (in cui il tono galante e melodrammatico si sposa con trascuratezza grafica e improvvisi scarti verso il basso), alle lettere a Francesco Algarotti (caratterizzate da un italiano più aulico e sorvegliato), all'affettuoso omaggio idiomatico a Carlo Goldoni (parzialmente in veneziano). L'《impetuoso italiano epistolare di Byron» - affascinante anche nella sua consapevole trascuratezza e nelle sue idiosincrasie (Cartago, 2002) - è invece lingua dell'amore. L'italiano di James Joyce è un felice impasto di registri linguistici e stili differenti (Melchiori, 1994).

9 Ciò è particolarmente evidente nell'italiano epistolare di Mozart, ampiamente caratterizzato «dalla freschezza e dalla grazia della spontaneità e dell'improvvisazione, fuori di ogni preoccupazione letteraria e normativa» e posto spesso e volentieri «sotto il segno dello sperimentalismo giocoso, del ludismo verbale e del plurilinguismo» (Folena, 1983: 435, 442).

${ }_{10}$ Sieghard Brandenburg, Beethoven Briefe, voll. 1-6, München, G. Henle Verlag (traduzione italiana a cura di Luigi Della Croce, Ludwig van Beethoven. Epistolario, Ginevra-Milano, Skira). L'edizione delle lettere numerate progressivamente - consta di sei volumi, divisi per intervalli di anni. Citeremo pertanto le lettere con il numero indicato nell'edizione. Ove necessario ai fini della ricerca sarà riportata anche la data. L'edizione dei Briefe consta di 2292 lettere, non tutte scritte da Beethoven, ma comprende anche epistole indirizzate al musicista, «in modo da ricostruire al massimo, delle attuali possibilità, il contesto sociale, economico ed artistico in cui il musicista ha operato e nel quale si è svolta la sua personalissima vicenda umana» (Prefazione alla versione italiana, p. XX). Indicazioni utili sulla tradizione del carteggio beethoveniano sono nell'Introduzione, p. 20. Cfr. anche Buscaroli (2020: 42).

${ }^{11}$ Cfr., per esempio, le lettere n. 457, 1644 (altre, per es. 208, 259, 515, non sono autografe).

12 Soprattutto negli incipit (liebster Baron Dreckfahrer je vous suis bien obligè [sic] pour votre faiblesse de vos yeux, $\mathrm{n}$. 35) e negli explicit (adie [sic], n. 34; adieu mon ami à bon marché, n. 39; adieu, n. 417; A monsieur monsieurn. n. 23; vous êtes invitè [sic], de diner chez moi, n. 1540). 
lettere interamente in italiano, indicate però dal curatore come «Original von fremder Hand, Unterscrift von Beethoven», quindi redatte da un'altra persona e soltanto sottoscritte dal musicista. Esse hanno in comune lo stesso destinatario, il collezionista ed editore musicale scozzese George Thomson. Si tratta delle lettere n. 178 (del maggio/giugno 1804), n. 739 (del 15 settembre 1814), n. 753 (di ottobre 1814). Nell'epistolario risultano altre lettere a lui indirizzate, ma scritte in francese di pugno del Maestro $^{13}$.

Detto ciò, sicuramente vale anche per Beethoven quanto indicato per Mozart da Folena (1983: 443), ossia la presenza di due registri comunicativi: una lingua familiare e affettiva e una lingua professionale e tecnica nel settore musicale; registri che, in non poche sue epistole, si presentano spesso mescolati.

\section{IL LESSICO MUSICALE}

Come dicevamo in apertura, non sorprende di ritrovare nella scrittura beethoveniana una messe molto nutrita di tecnicismi musicali italiani ${ }^{14}$.

Non possiamo, inoltre, non considerare il fatto che l'italiano è utilizzato da Beethoven anche nei titoli di alcune sue opere (pensiamo all'Eroica: Bonomi, 2015: 16), e nelle dediche (celeberrima quella a Napoleone Bonaparte ${ }^{15}$ ), fatto non nuovo ai musicisti tedeschi ${ }^{16}$.

Non solo. Gli inserti più lunghi in cui è usato l'italiano sono proprio quelli in cui il musicista impartisce istruzioni su come deve essere eseguito un brano o interviene con correzioni sul pezzo: [...] gleich anfangs: due Violoncello $<1$ mo $>$ Solo 1 mo e 2 do con sordino $<2 d o>$ gli violoncelli tutti coi bassi 370 'proprio all'inizio'; si ha s'imaginar la battuta di 6/8 (465, con la ridondanza pronominale); dopo il Maggiore Presto si ricomincia dal segno [...] il Minore 3/4, e continuendo si fa la seconda parte solamente una volta fin' à questa fermata; poi si prende subito la Coda 2200; per la Misura del Largo si conta nel largo sempre quattro semicrome cioè [... $]^{17} 1295$.

Come rilevato da Bonomi (2010: 224) la compresenza di prestito integrale e adattamento è una costante per il tedesco ${ }^{18}$, e la scrittura di Beethoven non fa per niente eccezione, ma in questa sede ci limiteremo ad un veloce elenco degli italianismi schietti ${ }^{19}$, per poi produrre qualche osservazione conclusiva. Essi sono distribuiti in diversi settori ${ }^{20}$;

${ }^{13}$ Cfr. le lettere numero 161, 167, 259, 457, 1085, 1244, 1248, 1303.

${ }^{14} \mathrm{La}$ quantità di italianismi musicali in tedesco è senz'altro maggiore che nelle altre lingue europee, cfr. Bonomi (2010: 218): «prima di tutto per fattori di ordine storico-musicale (massiccia e prolungata influenza della musica italiana, straordinario sviluppo della musica in Germania nell'800)»; una percentuale che «secondo gli studi, si attesta intorno al 60\%; seguono, a notevole distanza, le voci del lessico generale, e poi gastronomia, arte, teatro, commercio, economia, finanza, sport, religione e via via altri ambiti» (ibidem).

15 Poi sostituita dopo l'incoronazione imperiale. Cfr. Dahlhaus (1990: 38), Pestelli (2016), Morale (199: 125131). Cfr. Carli Ballola (1977: 63): «Il frontespizio della Terza Sinfonia titolata Bonaparte era stato furiosamente lacerato e la dedica originaria sostituita con una nuova, che suonava insieme condanna e rimprovero per tanti ideali infranti: Sinfonia eroica composta per festeggiare il sovvenire d'un grand'uomo».

16 Folena (1983: 437) ricorda la dedica in italiano di Mozart ad Haydn dei Sei quartetti. Da parte nostra aggiungiamo almeno la Sonata in Re maggiore detta All'imitatio Gallina cucca di Johan Sebastian Bach (BWV 963), in cui è citata una razza di galline veneta di cui quella bachiana è un'attestazione assai precoce (1704), essendo con ogni probabilità errata l'interpretazione avanzata da Fabbri (1964a: 159): «forse, nel suo italiano approssimativo, Bach voleva dire "della gallina e del cuculo"».

17 Segue l'indicazione delle note relative.

${ }^{18}$ La polimorfia continua è notata anche da Folena (1983: 466 alla nota 45) nella scrittura dei Mozart (padre e figlio).

${ }^{19}$ I tecnicismi sono riportati con l'iniziale originale che essi hanno nelle lettere. Abbiamo escluso dall'elenco quelli che compaiono in lettere non di pugno di Beethoven (cfr. per es. Andantino in una lettera scritta in francese, la 623, di mano di Oliva).

${ }^{20}$ Come osserva acutamente Bonomi (2010: 187), lo studio della terminologia musicale presenta non pochi problemi, alcuni di questi legati soprattutto all'eterogeneità degli àmbiti e delle categorie che investe. 
seguiremo (per quanto possibile) la classificazione indicata in Bonomi (2010). Abbiamo termini che sono legati:

- all'esecuzione, alla dinamica, alle indicazioni agogiche di movimento e di transizione ${ }^{21}$ : (ganz) a la cappella'(completamente) a cappella, senza accompagnamento strumentale'22 (1621; 2 occ., di cui una con l'iniziale maiuscola), adagio (54) (anche adagio cantabile, 393), allegretto (370) (e allegretto vivace, 1927), allegro (54) ${ }^{23}$ (anche allegro assai, 1927; allegro vivace, 393; allegro ma non troppo e ben marcato, 2022), andante (54) ${ }^{24}$ (anche andante molto cantabile, 1927; andante maestoso, 2223; andante moderato, 2223), arpeggio (393), bassi (370), basso ostinato (474), battuta (465), fermata 'pausa, sospensione della frase musicale' (2200), fortissimo (393), grave (31), (non) ligato 'modo di esecuzione di una linea melodica, senza interruzioni o stacchi tra una nota e l'altra' (1927), maestoso (1927), Maggiore (2200), [alla] marcia (2223) $)^{25}$, Minore (2200), mosso (1927), obbligato 'detto di parte strumentale che non si poteva omettere o sostituire durante l'esecuzione' 42 (anche in tutti obligati [sic], 4926), obligatissimo (2032), presto (465), prestissimo (465), ritardando 'rallentando' (380), Scherzoso (1309), segno (2200), semicrome pl. (1295), staccato (393), tempo (tempo allegretto, 548; tempo primo $\left.0^{27}, 1927\right)$, vivace (1315; anche molto vivace, 1315);

- teoria e composizioni: Ariette pl. $^{28}$ (1561), coda (10), fantasia ${ }^{29}$ 'tipo di composizione musicale' (233) (anche nel sintagma fantasia für pianoforte, 285), minuetto (54), operetta (451), oratorio 'forma musicale drammatica' $(152)^{30}$, partita 'forma musicale' (19), scher:o (393), Trio (380) e gran Trio (899);

- strumenti musicali (e loro parti): clarinetto (54), clavicembalo (93), ContraBasso (49), corno (49), fagotto (49), flauto [primo] (251), forte piano (23) (anche nella grafia forte-piano, 23), oboe (1857), piano 'strumento' (116), pianoforte 'strumento' (8; anche nelle varianti: pianoForte, 1071; Piano-forte, 1071; piano forte, 3) ${ }^{31}$, sordino (370), viola (49), violino (10), violoncello (370), voce (a tre voci, 73);

- canto e opera: libretto 'testo per musica32 (105), Mezzo soprano (1621), tenore (39).

Tutti i termini indicati (ad eccezione dell'avverbio obligatissimo e del sostantivo semicrome $^{33}$ ) sono registrati come italianismi in tedesco da DIFIT, ed in particolare possiamo segnalare che alcuni di essi compaiono nelle lettere di Beethoven prima dell'attestazione riportata dai repertori tedeschi (in alcuni casi la retrodatazione è notevole): corno $(1800 ; 1802)^{34}$, fugato $(1819 ; 1865)$, minuetto $(1801 ; 1806)$, mosso $(1825 ; 1876)$,

${ }^{21}$ Per alcuni interessanti aspetti della lingua delle partiture beethoveniane, cfr. Giardini (2014).

22 Inseriamo una breve definizione del tecnicismo solo nei casi in cui, non essendoci il contesto, si potrebbero creare delle ambiguità, tenendo presente che molti di essi ricorrono nelle indicazioni di Beethoven per l'esecuzione delle partiture. DIFIT registra sia la locuzione avverbiale a cappella, sia alla cappella.

${ }^{23}$ In alcune lettere anche in maiuscolo, cfr. lettera n. 1309.

${ }^{24}$ In alcune lettere anche in maiuscolo, cfr. lettera n. 993.

${ }^{25}$ La parola non è datata in tedesco da DIFIT.

${ }^{26}$ La stessa grafia in un'altra lettera Violonschell oblig[ato] 1278.

27 Per l'influenza che l'italiano ha avuto nell'impiego di questa informazione agogica, cfr. Giardini (2014: 220).

${ }^{28}$ Nella lettera riferito alle op. 121b, op. 122 e op. 128 (cfr. nota 3 a p. 36).

${ }^{29}$ Per l'impiego di questo termine, cfr. Giardini (2014: 223).

${ }^{30}$ La parola non risulta attestata in tedesco da DIFIT.

31 È piuttosto continua l'alternanza tra piano (meno frequente), pianoforte (più frequente, nelle sue varianti grafiche) e la parola tedesca Klawier (preponderante). Cfr. Bonomi (2015: 18 alla nota 28): «Pianoforte (1774), affiancato a meta del XIX secolo dall'abbreviazione Piano, è stato in seguito del tutto soppiantato da Klavier, utilizzato in generale per indicare strumenti a tastiera (anche clavicembalo)».

32 Per le diverse accezioni di questo termine in tedesco, cfr. Bonomi (1991: 208 e 2010: 220).

33 DIFIT registra soltanto Croma.

${ }^{34}$ La prima data è quella della lettera in cui compare la parola; la seconda quella indicata da DIFIT. 
piano $(1802 ; 1850 \mathrm{ca}$.$) , scherzoso (1819 ; 1879$; un interessante caso in cui l'accezione musicale non esiste in italiano $\left.{ }^{35}\right)$, tenore $(1798-99 ; 1800 \mathrm{ca}$.$) . Nella scrittura beethoveniana inoltre$ Ariette, ContraBasso e Mezzo soprano non sono adattati, mentre in tedesco sono attestate, stando alla documentazione del DIFIT, soltanto le forme integrate (Arietten, Kontrabaß e Merzosopran).

Un dato interessante è che Beethoven "de-tecnicizza" alcuni di questi lessemi utilizzandoli col valore semantico generale che essi hanno in italiano (e non in tedesco, come documenta il DIFIT che ne registra solo la valenza musicale ${ }^{36}$. Si tratta degli avverbi presto e prestissimo con valore temporale, come mostrano i contesti, numerosi e chiarissimi: bitte sogleich zu eiligst prestissimo 'per favore prestissimo subito in fretta' (967), Es eilt also presto Prestissimo 'quindi presto è urgente prestissimo' (967); Antwort auf meine Frage wegen der Ertmann noch heute baldigst (presto prestissimo) 'rispondi alla mia domanda su Ertmann oggi il prima possibile (presto prestissimo)' (1092), jedoch prestissimo 'comunque prestissimo' (1100), in Eil in Eil prestissimo 'di fretta, di fretta prestissimo' (1246), ersuche ich sie nochmals mir die Antwort auf Alles presto prestissimo zu senden 'ti chiedo di nuovo di inviarmi la risposta ad Alles presto prestissimo' (1365), eilt euch prestissimo mit allem 'sbriga tutto prestissimo' (2052); anche in chiusura di lettera: Prestissimo - Responsio il suo Amico ed Maestro (1302).

Proprio la "fretta" è uno dei leitmotiv della grammatica epistolare del Titano ${ }^{37}$. E gli avverbi citati non sono le uniche tessere italiane di questo campo semantico. Abbiamo infatti anche veloce (sondern veloce prestissimo 'piuttosto', 1302) e subito che è usato in una locuzione verbale, volti subito, un'istruzione musicale per indicare di girare velocemente la pagina dello spartito, ma che nei contesti individuati sembra indicare semplicemente un modo del mittente per segnalare al destinatario, di solito in chiusura di missiva, di svolgere quanto prima ciò da lui richiesto (per es. Volti subito mehr presto prestissimo 'di più', 993).

\section{IL LESSICO NON TECNICO}

L'italiano usato da Beethoven non presenta alcun carattere pedantesco, formale o letterario ed è accomunabile per tanti versi al ritratto che Folena (1983) traccia di quello mozartiano $^{38}$. Al di là dell'innegabile maggiore padronanza della nostra lingua da parte del maestro di Salisburgo, le somiglianze si riscontrano soprattutto in un uso giocoso e quasi comico dell'italiano (Folena, 1983: 433) ${ }^{39}$ e nel gusto per il pastiche linguistico (al tedesco sono accostati anche il francese, l'inglese, il latino, il latino maccheronico ${ }^{40}$.

\footnotetext{
${ }^{35}$ Cfr. Bonomi (2010: 189). Il fenomeno è tutt'altro che isolato in tedesco; Bonomi segnala anche amorevole, appenato, sereno (p. 218).

36 Bonomi (2010: 218) documenta il passaggio per i sostantivi Quartetto, Tempo, Duo.

${ }^{37}$ Numerose lettere si aprono o si chiudono proprio così: Eiligst u. Schleunigst (2152, 'con la massima fretta e velocità'), in Eil ihr Freund (2127, 'in fretta il suo amico'), Nur geschwinde Erinnerungen (1927, 'solo qualche avvertimento in fretta'). Cfr. anche questi contesti in cui sono usate espressioni latine: Cito. Citissime (2241), Angekommene Briefe werden gebeten citissime hieher zu schicken (972, 'le lettere che sono arrivate devono essere inviate qui citissime').

${ }^{38}$ Decisamente diverso è l'italiano di Voltaire, di spiccata matrice libresca, «costruito sulle grammatiche e i manuali italo-francesi del Settecento, con un pizzico di vocaboli e locuzioni colti dall'esperienza diretta della conversazione [...]» (Folena, 1983: 411); cfr. anche Meacci (2001: 449). Inoltre per Voltaire «[...] l'immagine della lingua e della cultura italiana è prevalentemente legata al melodramma» (Folena, 1983: 223).

${ }^{39}$ Lo studioso parla di «gusto giocoso del travestimento linguistico e dello spettacolo»; cfr. Hildesheimer (2006: 127) che parla di «spiccatissimo senso della comicità che raggiungerà [...] livelli diabolici». Cfr. anche Pestelli (1979: 233): «ha pure uno stile letterario tutto suo: una simpatia eccezionale per la jonglerie verbale e i doppi sensi [...] un gusto per le contraffazioni stilistiche, con prestiti dal gergo militare e dittatoriale, e tutta una gradazione di toni che vanno da un materialismo desunto da Shakespeare e Rabelais al piglio biblico di Klopstock».

${ }^{40}$ Cfr. Folena (1983: 434) che parla, con una formula davvero felice, di «concerto linguistico mozartiano».
} 
Ma passiamo all'uso di parole italiane non di stretta pertinenza musicale, poco più di una settantina, che Beethoven usa inserendole nel tessuto delle lettere. Sono soprattutto sostantivi (anche in locuzioni) e aggettivi: addio (111), (ch'a detto l') amato bene? (144), (con) amore (457; la lettera è in francese), (meine) asini (1034; in riferimento ai suoi collaboratori), carcere (1030, sogleich in Carcere bringen zu lassen, 'da portare immediatamente in carcere'); illustrissimi (911), mediocre (911), Penale (2192; 2 occ.), (senza) replica ${ }^{41}$ (457, in una lettera in francese), veto (1035), virtuoso (809; la chiusa è in inglese: Mr. Salomon ${ }^{42}$ most renowned virtuoso in the service of His Royal Highness the Prince Regent, anche pl., 1337), (Dukaten) a vista 'consegnati subito' (2154), (ultima) volta $\left(457^{43}\right)$; ma anche avverbi e congiunzioni: basta cosi (216 e 2057), usato come formula discorsiva di passaggio ad altro argomento ${ }^{44}$. Tra le forme verbali segnaliamo la coniazione beethoveniana cavateri 'attenzione' (949; Beschummelung her giehe, erst vom 3 ten Aug. etc nun Gott vergeltsnicht - aber büten muß man sichCavateri), commentata dal curatore Brandeburg «Verballhornung des lateinischen 'cavete', 'hütet euch'» ('corruzione del latino cavete 'attenzione' cfr. nota 3, p. 274 dei Briefe) ${ }^{45}$.

In alcune lettere abbiamo poi dei veri e propri code switching, come nei seguenti contesti: sie hat mir gestern ein Zeugnis übergeben, non hai denaro ('ieri mi ha consegnato una nota scritta, non hai denaro', 2131) e bald hoffe ich euch zu sehn ed a pagare $i$ Conti 'spero di vederti presto ed a pagare i conti', 1425).

Più frequentemente ritroviamo l'italiano nelle formule di apertura e chiusura delle lettere; avremo quindi espressioni formulari e titoli. Tra i più usati: amico (1302), barone (37, scherzosamente abbinato al corrispondente tedesco in una sorta di lungo richiamo: mein liebster Baron barone, baron! $)^{46}$, conte $(114$, Geliebtester conte di musica; conte ha due altre occorrenze all'interno della lettera), fratello (2024), maestro (1894), principe Proffessore (1343, riferito all'Arciduca Rodolfo), signore. Segnaliamo inoltre Bestes caro oggetto amabile 1919 e $A l$ Signore Milord stimatissimo Nominato Scuppanzig grand uomo della città da Vienna (1939, nella lettera a Ignaz Schuppanzigh che doveva eseguire il Quartetto n. 12 in Mi bemolle maggiore, op. 127).

Qualche precisazione. Amico è usato anche in dittologia amico und Conte di Musica (114), il suo amico ed maestro (1302).

Nella lettera a Franz Brunsvik (281, datata Vienna 11 maggio 1807), Beethoven "glossa" il pronome personale di prima persona plurale, wir, scrivendo amici: So oft wir (mehrere) (amici) Deinen Wein trinken, betrinken wir Dich, d.h. wir trinken Deine Gesundheit ('ogni volta che noi (molti) (amici) beviamo il tuo vino, ci ubriachiamo, cioè beviamo alla tua salute').

${ }^{41}$ Per questa indicazione formale, Giardini (2014: 231 alla nota 42) indica come frequente in Beethoven repetizione che non risulta nel nostro corpus.

42 Johann Peter Salomon (1745-1815), violinista e compositore tedesco, che si trasferì a Londra dove morì.

${ }^{43} \mathrm{Si}$ tratta di una lettera in francese a George Thomson: NB: Quand on prend l'ultima volta dans les airs Ecossais, on laisse 123 etc volta, c'est à dire, on ne sonne pas toute la Mesure de 123 etc volta.

${ }^{44}$ Cfr. la lettera 2057: «[...] leid thut es mir dich mit diesem alten teufel so lange wissen zu müßen halte nur immer fern von ihr - du must ihr einen Brief in meinem Nahmen an den Verwalter das spitals schreiben, wo du anführst, daß sie am ersten nicht gekommen zum Theil weil sie nicht wohl theils weil mehrere Menschen hieher zu mir gekommen - Basta così), in traduzione 'Mi dispiace pensare che tu debba stare per tanto tempo con quel vecchio demonio tieniti lontano da lei. A suo riguardo devi scrivere una lettera a mio nome all'amministratore dell'ospedale, dicendo che non è venuta il primo del mese perché non stava bene e perché varie persone erano in visita qui da me. - Basta così -'. Per l'uso di basta così col medesimo valore in Metastasio, cfr. Serianni (2005: 101) che ne segnala l'uso anche nell'epistolografia di inizio Ottocento (Antonelli, 2003).

${ }^{45}$ È interessante notare che nell'edizione in lingua inglese, la curatrice riporta la lezione cavatevi (p. 285), probabilmente in questo caso più corretta di quella indicata dall'edizione tedesca.

${ }^{46}$ La lettera è indirizzata a Nikolaus Zmeskall, giovane funzionario della cancelleria di corte, per tutta la vita uno dei più devoti e preziosi amici di Beethoven che nei suoi confronti «nei momenti di buona luna scatena una sua rozza euforia fatta di giochi di parole e di grossolane lepidezze» (Carli Ballola, 1977: 32). 
Il sostantivo è però usato di preferenza in latino, lingua che, come vedremo, ha un certo spazio nella scrittura beethoveniana. Da solo: amicus (1633) (anche seguito, in chiusa di lettera, dal suo cognome ${ }^{47}: 1380 ; 8$ occ.), o in costruzioni più complesse (wie immer dero Bester amicus ad amicum de amico 'come sempre il migliore' (1065), amicus fidelis (2049), optime amice (2158), clarissime amice (1209), Sehr B [e]ster optimus optime (1524), amice optime (1588), Amicus optimus (1588), Amicus ad Amicum (1266).

Fratello e signore sono usati spesso in coppia (e il binomio ricorre più volte anche in tedesco, Herr Bruder, 1978; H. Bruder, 2024): Sig. Fratello (2007, in una lettera al nipote Karl), Signore Fratello (2221, in una lettera a Tobias Haslinger). In queste lettere gli appellativi che Beethoven utilizza in riferimento al fratello minore Johann, in cortocircuito tra loro, rappresentano uno dei segni della scarsa stima e considerazione che il musicista aveva di lui ${ }^{48}$.

Già Nohl (1877: 31-32) segnalava Kain 'Caino', Gebirnfresser 'mangiatore di cervelli', Judas Ischarioth 'Giuda iscariota'. E ancora: Schurkische Bruder ('fratello malvagio', 1978), Herzlosen ('senza cuore', 1991), unbrüderliche Bruder ('non fraterno fratello', 1980) e, con il ricorso al prefisso spregiativo pseudo, Pseudo-Bruder (2023), Hr. Bruder (Pseudo) (2035), [...] du bist swar auch ein lumpenkerl ein Pseudo bruder'a dire il vero anche tu sei un furfante e uno pseudofratello' (2024). L'italiano concorre con lo spregiativo Asinaccio (H[err] Bruder Asinaccio hat geschrieb 'mi ha scritto quell'asinaccio di mio fratello', 1996; in Asinaccio's Wobnung 'nell'alloggio dell'asinaccio' (2070).

Signore inoltre ricorre in altri contesti interessanti, in cui è possibile rinvenire il gusto del compositore per lo scherzo. Appella l'amico Schindler Signore Papageno (1684; anche per il signore povero Papageno, 1654; per il Signore Nobile Papageno Schindler, 1684; anche in francese Pour Monsier Papageno, 1680), dal nome del curioso personaggio del Singspiel Die Zauberflöte di Mozart, un uccellatore girovago, con gli abiti coperti di piume, un sempliciotto, buffo ed eccentrico. Il compositore e violinista italiano Giovanni Battista Polledro viene appellato signore polledrone (591).

Tra gli italianismi impiegati da Beethoven, appena un terzo è registrato in tedesco dal DIFIT con la medesima forma e significato (tra cui addio, basta, contento, dolce, principe, replica, senza, virtuoso, volta); altri (conto, illustrissimi) sono accolti, ma nella forma adattata (Konto, Illustrissimus) o, come per generalissimo, nella contaminazione Generalissimus.

Altri lessemi presentano significati diversi da quelli indicati dal repertorio (amabile e prestissimo che sono registrati solo col significato musicale; denaro, nel repertorio 'dodicesima parte del soldo', mentre nelle lettere ricorre col significato generico di 'soldi'; bene risulta soltanto come interiezione e non come sostantivo).

Di altri ancora le lettere beethoveniane presentano precoci attestazioni: (Dukaten) a vista 'consegnati subito' (1826; 1806), con amore $(1810 ; 1806)$, signore $(1823 ; 1806)$ o consentono la retrodatazione: conte (1795ca.; 1879), maestro (1824; 1901).

\section{I GIOCHI DI PAROLA CON IL SIGNIFICATO DEI COGNOMI}

Beethoven non è nuovo a giochi di parole e calembours e lo bumour è presente nel suo epistolario senza soluzione di continuità (Van der Borren, 1927: 109). Il suo gusto per lo scherzo e la battuta è evidente, e si avvale di almeno due modalità di espedienti linguistici.

\footnotetext{
47 A volte anche con "latinizzazione" del complemento di origine: Euer amicus Beethoven Bonnensis (1444).

${ }^{48}$ Bersaglio anche di ironia, come nel seguente passo che allude alle sue fortune economiche: Bestes bruderl. Besitzer aller Donauinseln um Krems. Director der gesammten Osterreichischen Pharmacie ('Miglior fratello. Proprietario di tutte le isole del Danubio intorno a Krems. Direttore dell'intera farmacia austriaca', 1505).
} 
Innanzitutto, nell'uso di accostamenti antonomastici (come Papageno che abbiamo visto usato in relazione a Schindler, ma non si tratta di un caso isolato). Alla Baronessa Dorothea von Ertmann (sua affezionata allieva di pianoforte e ottima esecutrice delle sue opere) si rivolge chiamandola Cecilia, il nome della santa protettrice della musica (1093) e in apertura di lettera (Sehr Bester Falstaff, 1379), apostrofa Falstaff Carlo Boldrini, collaboratore della casa editrice musicale Artaria, per via delle sue fattezze fisiche che richiamavano il personaggio shakespeariano, un cavaliere grasso e vanaglorioso ${ }^{49}$.

L'altra soluzione consiste nei numerosissimi giochi di parola che egli produce in relazione ai significati dei cognomi e all'assonanza di alcuni di essi con altre parole, in una sorta di gioco paronomastico continuo ${ }^{50}$. Solo qualche esempio. Si riferisce all'amico Peter Joseph Simrock con l'appellativo überrock 'cappotto; impermeabile' per via dell'assonanza tra le due parole (Mein bester Überrock ihres Hr. Papas!, 977). Il compositore ed editore Anton Diabelli è accostato a diavolo: con l'espressione Diabolus Diabelli gli si riferisce in alcune lettere a Sigmund Anton Steiner (808, 814, 847). Apre una lettera (2050) a Joseph Linke, un violoncellista, esecutore di alcuni suoi quartetti, giocando con il suo cognome, che in tedesco significa 'sinistra', con la formula Lieber Linke und rechte ('Cara sinistra e destra').

Ma è l'amico, aiutante e segretario Karl Holz ${ }^{51}$ ad essere il più bersagliato, per via del significato del suo cognome, 'legno'. Beethoven si sbizzarrisce con creazioni tra tedesco e latino: Bestes Holz. Christi (2193, 'ottimo legno di Cristo'), Holz Schaffe Holz! (2175, 'Holz porti della legna, ${ }^{52}$ ), Bester Span! Bestes Holz Christi! (2028, 'Ottimo truciolo. Ottimo legno di Cristo'), dem Holz christi oder dem span des Holz Christi (2012, '[non avrei voluto troppo fidarmi] del legno di Cristo o di un truciolo di legno di Cristo'), Bestes Maba [g]oni Holz! Cedern sind unß nicht bekannt nehmt vorlied (2043, 'ottimo legno di mogano, il cedro ci è sconosciuto, dovrete accontentarvi'), Bestes lignum crucis (2080, una citazione da Ecce lignum crucis, un canto della liturgia del venerdì santo).

Beethoven arriva a coniare il verbo pertobiassen sul nome di Tobias Haslinger: [...] ja, ja Tobias bleibt ein T[obias] wir wollen ihn aber doch noch pertobiassen (2043, 'sì, sì Tobias sarà sempre un T[obias], ma noi vogliamo ancora più tobiassarlo') e l'aggettivo Miserablatzin: dem Miserablatzin schmeichle ja nicht (2008, al nipote Karl 'non adulare quel Miserablatzin'), in cui all'aggettivo miserable aggiunge la sillaba -tzin (così spiega l'editore: cfr. nota 6 a p. 107), alludendo così al Principe Galitzin, un violoncellista dilettante e suo ammiratore con cui aveva avuto degli screzi.

L'ironia non si limita agli altri, ma investe volentieri anche lo scrivente stesso. Tutt'altro che rari i casi in cui Beethoven usa espressioni buffe ${ }^{53}$ per abbassarsi agli occhi dei propri corrispondenti, per esempio firmandosi Beethoven minimus (140, a Zmeskall) ${ }^{54}$, Beethoven Miserabilis (624, a Zmeskall), Tantus quantus lumpus (1172; 'specie di mascalzone',

49 Secondo Carli Ballola (1977: 43) il nomignolo Falstaff è usato anche in riferimento al violinista Schuppanzigh «per la sua abbondevole figura».

50 Per l'uso di questo espediente da parte di Mozart, cfr. Folena (1983: 433-34).

${ }^{51}$ Holz è benevolmente preso in giro anche con espressioni come: Bester Herr u. Luccullischer Küchenprocurator (2081, 'Ottimo signore e luculliano procuratore delle cucine': nella lettera Beethoven chiede all'amico di comprargli un luccio per il pasto).

52 Per la traduzione di questo passo, come dappertutto, si segue quella di Luigi Della Croce, ma il significato letterale è 'legna crea legna'.

53 Carli Ballola (1977: 41) riporta lo stralcio di una lettera destinata all'amico Johann Nepomuk Hummel, suo amico e compagno di studi alla scuola di Albrechtsberger in cui il musicista si firma Mehlschöberl, dal «nome di un cuoco, personaggio di una commedia popolare molto in voga a quel tempo: uno dei molti nomignoli che si affibbiava [...] nei momenti di buonumore, e che alludeva forse alla sua abitudine di improvvisare agli amici certi pranzetti non propriamente succulenti, conclusi da un altrettanto problematico "caffè alla Beethoven"».

54 Anche Mozart si diverte a giocare con la traduzione del suo nome, cfr. Folena 1983: 434. Beethoven traduce il suo nome anche nelle lettere in francese (cfr. per es. le lettere n. 457 e 1644). 
probabilmente un adattamento latinizzato del tedesco lump 'cencioso, miserabile': Van der Borren, 1927: 110), senza dimenticare l'onnipresente generalissimo (511; una cinquantina di occorrenze) ${ }^{55}$, nelle lettere che egli scrive all'editore Sigmund Anton Steiner (anche latinizzato: generalissimus, 805; e in forma abbreviata: $\operatorname{der} g \_s$, cfr. per es. 985$)^{56}$; ma anche Generallieutenants ('tenente generale' a Tobias Haslinger, cfr. per es. 891).

L'uso del latino per celia ${ }^{57}$ è tutt'altro che isolato: addio vortrefflicher plenipotentiarius Regnis Beethovensis (111, 'più eccellente'), Secretarius perpetuus (1922, riferito al nipote Karl). Ma anche nel tradurre il nome del destinatario delle lettere (in questo caso, l'amico, scrittore e librettista Joseph Karl Bernard): Dominus Bernardus non sanctus (1588), Bernardum non santum (1587), Bester Bernardus non Sanctus (1306), Lieber Bernardus non sanctus (1340).

In due casi poi usa per sé l'appellativo povero musico: ich bin wie immer un povero Musico (970, 'come sempre, sono un povero musico'); anche giocando col significato che in tedesco aveva la parola, ossia 'castrato': [...] denn ich bin ein armer österreichischer Musikant povero Musico! Jedoch nicht im <Kastratensinn> (588, 'perché sono un povero musicista austriaco (ma non nel senso castrato)'.

\section{FRASI FATTE E CITAZIONI TRA ITALIANO, LATINO E TEDESCO}

L'italiano (come pure il latino) ricorre non solo in tessere isolate o in brevi formule di saluto, ma anche in aforismi ed espressioni proverbiali o aneddotiche. Qualche esempio. L'espressione anche io sono pittore (1306), frase tradizionalmente, ma quasi certamente con errore, attribuita a Correggio (cfr. Fumagalli, 1995: 221-22), è registrata da DIFIT (anch'io sono pittore, attestata nel 1879): l'attestazione beethoveniana consentirebbe di retrodatare la circolazione dell'espressione di non poco: al 1819). Proviene invece con molta probabilità dall'opera mozartiana Le nozze di Figaro, l'espressione $A$ b o dolce contento $(1385)^{58}$.

Non mancano frasi di pugno dello scrivente che assumono aspetto volutamente sentenzioso, come la musica merita d'essere studiata $(1091$; 1292) e questo è un piacere per un autore (85).

Anche le citazioni in latino ${ }^{59}$ contribuiscono alla particolarità ed eccezionalità dei Briefe beethoveniani ${ }^{60}$. Come nulla dies sine linea (1885 e 2236), di pliniana memoria, ad indicare la necessità dell'esercizio giornaliero (come dico sempre si legge nella lettera 2236; cfr. Fumagalli, 1995: 331).

Degne di nota in particolare le sue personalissime riformulazioni. Il celeberrimo detto attribuito a Giulio Cesare (Hier muß es heißen) veni vidi vinci!!! (1349, in chiusa di lettera, 'va

\footnotetext{
55 Per l'uso che Beethoven fa del sottocodice militare, cfr. Pestelli (1979: 233).

56 A cui Beethoven spesso aggiunge ulteriori annotazioni "personali”; cfr. per es. im Bett ('a letto', 985), in Donner u. Blitz ('in tuoni e fulmini', 1111).

${ }_{57}$ Per l'uso di parole e locuzioni latine come mezzo espressivo nelle cosiddette lettere "facete" o burlesche, cfr. Matt (2014: 266).

58 Al dolce contento (atto terzo, scena quinta).

${ }^{59}$ In alcuni casi lo scrivente commette degli errori. Sbaglia il caso in ipse miserum (414, dovrebbe essere all'accusativo: ipsum) o sostituisce un quam con com in parvum talentum com ego? (157); cfr. anche la dicitura deux ex machina (492).

${ }^{60}$ Numerose le parole e le locuzioni latine. Soprattutto nei saluti (gratias agimus tibi domine (1078); vale (186); vale et fave (2097), ma anche singole espressioni disseminate all'interno del dettato: raptus (62), memento (619), supplicandum (635), in loco (59), et sine mea culpa (1894), memento mori (2205), pax vobiscum (59), pia desideria (298), pro primo (49), satis est (465), Secula seculorum (1826), stante pede 'immediatamente' (1181), summa summarum, Nichts! Nichts! Nichts! 'in conclusione, niente! niente! niente!' (196), vita brevis, ars longa (1651), ut dicunt (1950), pleni gratia (920). Segnaliamo infine il tecnicismo ad libitum 'nelle didascalie, lascia all'esecutore libertà interpretativa' (10; varie occorrenze, es. erstere zwey Stimmen ad libitum, 1388; 'le prime due voci ad libitum').
} 
detto qui' in cui il musicista festeggia un risultato ottenuto). Anche la locuzione latina intelligenti pauca è rivisitata in sapienti pauca (1313; 4 occ.).

La locuzione dell'Andria di Terenzio (obsequium amicos), veritas odium parit 'la verità attira l'odio' è modificata in veritas non odium parit (1315 e 1773) o anche ich hoffe Veritas non odium parit ('spero che', 2097) in cui il non è sottolineato.

Dello stesso tenore la rivisitazione della formula attribuita a Catone (l'ozio è il padre dei vizi) in otium est vitium (1759).

Segnaliamo, infine, l'espressione, lapidaria, dixi (370, 2217, 1922: Dixi Beethoven), usata da Beethoven quando è preso da indignazione o ira: nei contesti indicati ricorre dopo una lunga domanda retorica che si apre con l'invocazione in nome del cielo e nella seconda vi è una lunga reprimenda nei confronti di Zmeskall. In un'altra lettera (1158) compare come chiusa in cui accusa benevolmente Schindler di non presentarsi ai suoi inviti; in un'altra (1312) è alla fine di una lunga e meticolosa serie di correzioni.

\section{L'INVETTIVA E IL TURPILOQUIO}

Che Beethoven non avesse un buon carattere è ampiamente (forse troppo) riconosciuto dai suoi biografi di tutti i tempi ${ }^{61}$ e i Briefe non mancano di documentarlo con una certa ampiezza. Spesso si tratta di commenti a metà tra il benevolo e lo scherzoso ${ }^{62}$, come caput confusum (riferito al nipote nella lettera 1745, in cui ritorna l'uso del latino per celia), Für Hrv Schinder Mährischer Schädel (1745, 'Per il sig. v Schindler zuccone moravo'), Bestes lümperl (2026) e Liebes lümperl (2042) 'caro bricconcello' nelle lettere al nipote Karl.

Un vasto stuolo di improperi è rivolto alla sua governante, Barbara Holzmann: Alten 'vecchia', Hexe 'strega' (cfr. le lettere 2070 a Karl e 2024 al fratello Johann), Satanas (2034 e 2043), die alte ganß 'la vecchia oca' (2012), alten teufel 'vecchio demonio' (2057); anche con ricorso a procedimenti litotici: Ungefährlichste aller Personen 'la meno pericolosa delle persone' $(2175)^{63}$.

Ma non mancano espressioni più forti, come Hr. Scheiserl (2024, 'signor Stronzo', riferito a Schindler), Schreib-Sudler! Dummer Kerl! (1953, imbrattacarte pasticcione! pezzo di cretino!' a Ferdinand Wolanek, un copista che aveva commesso degli errori grossolani, tanto che il musicista cita una frase di Plutarco ${ }^{64}$ nella lettera: als wenne die Sau die Minerva lehren wollte 'è proprio come se la scrofa volesse insegnare a Minerva') ${ }^{65}$.

${ }^{61}$ Cfr. Aprile (2020) «[...] la sua iconografia, così vicina al prototipo romantico dell'artista combattuto, tenebroso, morto in povertà, ha molto concorso alla sua popolarità presso il grande pubblico. E così la sua "testa leonina ben nota a tutti i viennesi" si attacca bene con un paio di giudizi forse impietosi nelle intenzioni, uno di Goethe ("è un selvaggio") e uno di Cherubini (che definisce la sua casa "una tana per gli orsi”)». E ancora, da Fabbri (1964: 7-8): «Tutti gli amici conoscono bene le sue collere improvvise, le ribellioni indomite, i modi spesso sconcertanti per l'assenza assoluta, in lui, di riguardi alle buone maniere del vivere mondano», pp. 7-8. Di «raptus improvvisi e irrefrenabili» parla Carli Ballola (1977: 42); di «abissi di depressione, intemperanza emotiva, stravaganze, sbalzi di umore» Pestelli (1979: 231).

${ }^{62}$ Cfr. anche il commento su Schlesinger in una lettera al nipote Karl (2054): entre nous il est pourtant juif'detto tra noi resta sempre un ebreo'.

${ }^{63}$ Cfr. Carli Ballola (1977: 66): «la descrizione del ménage domestico, se pure così si può chiamare, del maestro è sempre stata la parte più pietosa, e insieme più divertente delle biografie beethoveniane». È noto infatti il continuo succedersi di cuoche, cameriere, donne di servizio, governanti che non resistono a lungo con un padrone dalle abitudini impossibili.

${ }^{64}$ Cfr. Pestelli (1979: 232): «non si contano nei "quaderni” [di conversazione] e nelle lettere citazioni più o meno esplicite di Schiller, Goethe, Kant, Rousseau, Plutarco».

${ }^{65}$ Ecco la traduzione della lettera dopo l'incipit: «Corregga piuttosto gli errori dovuti alla Sua spavalderia frutto d'ignoranza, alla Sua presunzione e alla Sua stupidità, questo Le conviene meglio che volermi fare la lezione perché è proprio come se la scrofa volesse insegnare a Minerva». Dopo la firma aggiunge una postilla: «Era già stato deciso ieri e ancor prima di ieri, di non farLa più scrivere per me. Faccia l'onore a 
Scende decisamente al livello del turpiloquio l'incipit della lettera 1337 (dell'ottobre 1819, indirizzata alla casa editrice Artaria), Sehr BeSte Virtuosi senza cujonil: Beethoven richiede sdegnato sei copie di due sue opere e mostra i suoi dubbi sul fatto che l'editore si stia comportando correttamente, nel rispetto della legge. La parola (benché glossata con l'italiano coglioni nell'edizione spogliata ${ }^{66}$ ) e pur comparendo in un lacerto in italiano potrebbe risentire, come indica DIFIT (che in tedesco ne registra soltanto l'adattamento Kujon), e come suggerirebbe la presenza della vocale $u$, del francese couillon (a sua volta un italianismo ${ }^{67}$, anche se la marca plurale in - $i$ è certamente italiana.

\section{I CARATTERI LINGUISTICI DELL'ITALIANO DI BEETHOVEN}

La scrittura beethoveniana - fondamentalmente riconducibile all'uso settecentesco presenta svariate incertezze. Sul piano ortografico è molto frequente l'oscillazione nell'uso delle maiuscole per i sostantivi all'interno di frase - come in tedesco -: Violoncello/violoncelli; Largo/largo; Cappella/cappella, piano-Forte/Piano-forte (all'interno della stessa lettera) / piano forte, ecc.; ma sono poi spesso omesse nei nomi di persona: oliva (1315), pasqualati (224), giannattasio (1039), paisiello (519).

Piuttosto incerta si mostra poi la fusione o la divisione delle parole: in primis nell'uso dell'apostrofo, per esempio con troncamenti lasciati in sospeso (grand uomo). Oscillazioni si hanno anche nella grafia delle preposizioni, a volte assimilate all'articolo (cor), a volte staccate come nell'uso letterario (a la) e nella resa di parole composte (forte piano/ fortepiano; pianoforte/piano-Forte/piano forte).

Segnaliamo inoltre l'uso della preposizione a accentata (fin' à questa fermata) e l'ipercorrettismo nell'impiego della $d$ eufonica (ed Maestro).

A livello fonologico, possiamo notare nel vocalismo la chiusura di ligato ${ }^{68}$ ('legato'), l'ipergeminazione in Proffessore ${ }^{69}$ e lo scempiamento in obligati ${ }^{70}$ e obligatissimo (ma anche obbligato), mentre in ContraBasso la mancanza della doppia non sorprende trattandosi di una parola composta.

Per la morfologia soltanto l'uso errato dell'articolo plurale davanti a fricativa (gli violoncelli; ma cfr. tutti i violoncelli 1966) e la mancata concordanza in due Violoncello.

La maggior parte della corrispondenza beethoveniana è una felice mistura fra natura privata e natura lavorativa. I Briefe hanno, inoltre, perlopiù un carattere pratico, volto ad ottenere uno scopo preciso: al tempo di Beethoven la lettera corrispondeva ad una specie di «conversazione scritta con una o più persone assenti» (Introduzione, p. 17). Da qui anche il loro essere scritti «in modo molto impulsivo» (Introdurione, p. 38), a volte anche senza rispettare le norme della scrittura epistolare ${ }^{71}$. Il loro stesso aspetto esteriore (si pensi alla grafia che cambia direzione e dimensione, alla disposizione del testo spesso irregolare, all'uso iperbolico della sottolineatura per dare enfasi alle parole, alla messa in risalto di alcune espressioni mediante linee verticali e doppi punti) «tradisce le emozioni e le tensioni

Mozart e Haydn di non essere menzionati da Lei». Le sottolineature sono originali e l'ultimo pronome personale risulta sottolineato ben sette volte (cfr. nota n. 3 alla lettera).

${ }_{66}$ È indicata come corrispondente della parola coglioni anche nell'edizione inglese delle lettere curata da Anderson (1961: 845; indicata come lettera n. 975).

${ }^{67}$ In questo significato triviale dal 1813, Stendhal, TLF $i$.

${ }^{68} \mathrm{Il}$ tratto è ampiamente registrato da Tomasin (2009) nella scrittura di vari autori sette-ottocenteschi: Vico

(p. 37), Giannone (p. 89), Gozzi (p. 136). Per questa lezione, cfr. Giardini (2014: 230, nota 36).

${ }^{69}$ Tratto comune anche alla scrittura mozartiana, cfr. Folena (1983: 463, nota 28).

${ }^{70}$ La grafia obligati è anche nelle lettere di Mozart, cfr. Folena (1983: 463, nota 28).

${ }^{71}$ Cfr. Introdurione, p. 17. 
che determinavano lo scrivente» (Introdurione, p. 38). In questa architettura epistolare, l'uso dell'italiano, pur nella sua scarsa consistenza, è una delle cifre testuali che contribuiscono a rendere le lettere importanti testimonianze del vissuto del grande Musicista e rappresenta un ulteriore addendo della sua comunicazione spontanea, sospesa e altalenante tra il divertito, l'adirato, il malinconico. Una comunicazione ad ogni modo di carattere non formale, ma intima e confidenziale.

\section{RIFERIMENTI BIBLIOGRAFICI}

Anderson E. (1961), The letters of Beethoven, MacMillian Press, London (in tre volumi).

Antonelli G. (2003), Tipologia linguistica del genere epistolare nel primo Ottocento, Edizioni dell'Ateneo, Roma.

Aprile M. (2020), Il titano "beethoveniano", in Enciclopedia Treccani: https://www.treccani.it/magazine/lingua_italiana/speciali/Beethoven/01_Aprile. html.

Argenziano R. (a cura di) (2019), Jan Brueghel il Vecchio: le lettere in italiano dell'Ambrosiana. Edizione commentata, Biblioteca Ambrosiana, Milano.

Bonomi I. (1991), "Sul lessico del canto nel Settecento", in Rendiconti dell'Istituto lombardo Accademia di Scienze e Lettere. Classe di Lettere e Scienze Morali e Storiche, CXXIV, pp. 197-213.

Bonomi I. (1998), Il dolce idioma. L'italiano lingua per musica, Bulzoni, Roma.

Bonomi I. (2010), "La penetrazione degli italianismi musicali in francese, spagnolo, inglese, tedesco", in Studi di Lessicografia italiana, XXVII, pp. 185-235.

Bonomi I. (2015), "Italianismi musicali nel mondo", in Bonomi I., Coletti V. (a cura di), L'italiano della musica nel mondo, Accademia della Crusca, Go ware, Firenze, pp. 1030.

Buscaroli P. (2020), Beethoven, Mondadori, Milano.

Brandenburg S. (1996), Beethoven Briefe, voll. 1-6, G. Henle Verlag, München (trad. it. a cura di Della Croce L., Ludwig van Beethoven. Epistolario, Skira, Ginevra-Milano 19992005).

Brugnolo F. (1983), Plurilinguismo e lirica medievale. Da Raimbaut de Vaqueiras a Dante, Bulzoni, Roma.

Brugnolo F., Orioles V. (a cura di) (2002), Eteroglossia e plurilinguismo letterario (vol. $1^{\circ}$, L'italiano in Europa, Atti del XXI Convegno interuniversitario di Bressanone, 2-4 luglio 1993; vol. 2, Plurilinguismo e letteratura, Atti del XXVIII Convegno interuniversitario di Bressanone, 6-9 luglio 2000), Il Calamo, Roma.

Brugnolo F. (2009), La lingua di cui si vanta Amore. Scrittori stranieri in lingua italiana dal Medioevo al Novecento, Carocci, Roma.

Brugnolo F. (2011), "Scrittori stranieri, italiano degli”, in Enciclopedia dellitaliano, Istituto della Enciclopedia italiana, Roma, pp. 1290-1295.

Bruni F. (1999), "Dante e Byron: un incontro ravennate", in Letture classensi. Momenti della fortuna di Dante in Emilia e in Romagna, 28, pp. 95-153.

Carli Ballola G. (1977), Beethoven. La vita e la musica, Edizioni Accademia, Milano.

Cartago G. (2002), "Le lettere dall'Italia e le lettere in italiano di Byron”, in Brugnolo F., Orioles V. (2002), vol. 1º pp. 219-231.

Dahlhaus C. (1990), Beethoven e il suo tempo, (trad. it. di Dallapiccola L.), E.D.T., Torino.

DEUMM = Dizionario enciclopedico universale della musica e dei musicisti. Le biografie, diretto da Basso A., vol. I, UTET, Torino, pp. 375-414. 
DIFIT = Stammerjohann H. et al. (a cura di) (2008), Diz̨ionario di italianismi in francese, inglese, tedesco, Firenze, Accademia della Crusca, Firenze.

Fabbri $(1964 a)=$ Storia della musica Fabbri, vol. 2., Bach e il Settecento strumentale, Fabbri, Milano.

Fabbri (1964b) = Storia della musica Fabbri, vol. 4., Beethoven e il primo romanticismo, Fabbri, Milano.

Folena G. (1983), "L'italiano di Mozart nel concerto europeo del suo epistolario", in Id, L'italiano in Europa. Esperienze linguistiche del Settecento, Einaudi, Torino, pp. 432-469.

Fumagalli G. (1995), Chi l'ba detto? Tesoro di citazioni italiane e straniere, di origine letteraria e storica, ordinate e annotate da Giuseppe Fumagalli, Hoepli, Milano.

Giardini M. (2014), "La lingua italiana nelle partiture musicali fra XVIII e XX secolo", in Italiano Lingua Due, 6, 2, pp. 217-240:

https://riviste.unimi.it/index.php/promoitals/article/view/4698.

Hildesheimer W. (2006), Mozart, Rizzoli, Milano.

LESMU = Nicolosi F., Trovato P. (a cura di, con la collaborazione di Di Benedetto R.) (2007), Lessico della letteratura musicale italiana 1490-1950, Franco Cesati Editore, Firenze.

Matt L. (2014), "Epistolografia letteraria”, in Antonelli G., Motolese M., Tomasin L. (a cura di), Storia dellitaliano scritto. II. Prosa letteraria, Roma, Carocci, Roma, pp. 255282.

Meacci G. (2001), "L'italiano degli stranieri illustri tra il XVII e il XX secolo", in Serianni L. (a cura di), 2001, Società Dante Alighieri, Roma, pp. 445-453.

Melchiori G. (1994), Joyce. Il mestiere dello scrittore, Einaudi, Torino.

Morale U. (1999), Introduzione a Beethoven, Mondadori, Milano.

Motolese M. (2001), “L'italiano all'estero. Introduzione”, in Serianni L. (a cura di), 2001, pp. 439-441.

Nohl L. (1877), Beethoven's letəte Jahre, Ernst Julius Günther, Leipzig.

Pestelli G. (1979), L'età di Mozart e di Beethoven, Storia della musica ( a cura della Società Italiana di Misicologia), Vol. 6, Torino, EDT, Torino.

Pestelli G. (2016), Il genio di Beethoven: Viaggio attraverso le nove Sinfonie, Donzelli, Roma.

Rossi F. (2011), "Musica e lingua", in Enciclopedia dell'Italiano, Istituto della Enciclopedia Italiana, Roma, pp. 933-935:

https://www.treccani.it/enciclopedia/musica-e-lingua_\%28Enciclopediadell $\% 27$ Italiano $\% 29 /$.

Serianni L. (a cura di) (2001), La lingua nella storia d'Italia, Società Dante Alighieri, Roma.

Serianni L. (2005), "Libretti verdiani: quel che resta di Metastasio", in Tonani E. (a cura di), Storia della lingua italiana e storia della musica nel melodramma e nella canzone. Atti del IV convegno dell'Associazione per la Storia della Lingua Italiana (Sanremo, 29-30 aprile 2004), Franco Cesati Editore, Firenze, pp. 91-104.

Stammerjohann H. (1990), "L'immagine della lingua italiana in Europa", in Lo Cascio V. (a cura di), Lingua e cultura italiana in Europa, Le Monnier, Firenze, pp. 11-34.

Stammerjohann H. (1997), "L'immagine della lingua italiana in Europa", in Renzi L., Cortelazzo M. A. (a cura di), La linguistica italiana fuori d'Italia. Studi, Istituzioni, Collana Società di Linguistica Italiana 38, Bulzoni, Roma, pp. 27-51.

Stammerjohann H. (2013), La lingua degli angeli, Accademia della Crusca, Firenze.

TLFi $=$ Trésor de la langue française informatisé, Centre National de la Recherche Scientifique, Université de Nancy-2, Paris: http:/ /atilf.atilf.fr/.

Tomasin L. (2009), «Scriver la vita». Lingua e stile nell'autobiografia italiana del Settecento, Franco Cesati Editore, Firenze. 
Van der Borren C. (1927), "Beethoven e Orlando di Lasso", in Il pianoforte, pp. 108-111: http:/ / digitale.bnc.roma.sbn.it/tecadigitale/giornale/TO00191023/1927/unico/0 0000126.

Vitale M. (1982), “Correnti linguistico-culturali e problemi di lingua nell'Italia del primo Ottocento e la posizione di Stendhal", in AA. VV., Stendhal e Milano. Atti del XIV Congresso internazionale stendhaliano (Milano 19-23 marzo 1980), Olschki, Firenze, pp. 225-262.

Zanotti S. (1999), "Per un ritratto dell'artista italianato: note sull'italiano di James Joyce con edizione di un testo", in Studi linguistici italiani, 25, pp. 16-63. 